\title{
TRANSTHORACIC VERSUS TRANSHIATAL ESOPHAGECTOMY: COMPARATIVE STUDY REGARDING SURGICAL APPROACH IN ESOPHAGEAL CANCER
}

\author{
B. Filip ${ }^{\bowtie}$ I. Huţanu, I., Cristina Croitoru, Maria Gabriela Aniţei, \\ I. Radu, V. Scripcariu \\ University of Medicine and Pharmacy „Gr.T. Popa” Iaș i \\ First Surgical Oncology Unit, Regional Institute of Oncology Iaşi, Romania
}

\begin{abstract}
TRANSTHORACIC VERSUS TRANSHIATAL ESOPHAGECTOMY: COMPARATIVE STUDY REGARDING SURGICAL APPROACH IN ESOPHAGEAL CANCER (Abstract): BACKGROUND: Surgical resection can offer the best curative treatment for oesophageal cancer but is associated with high postoperative morbidity rates. Most common surgical approaches are transthoracic (TT) and transhiatal (TH) techniques. Transhiatal approach has the advantage of reducing the pulmonary morbidity in patients with impaired pulmonary function. AIM: The aim of this study is to compare the TT and TH approach, in terms of preoperative assessment and short term outcomes. MATERIALS AND METHODS: We performed an observational study on a prospective collected database which included all the patients diagnosed with oesophageal cancer in which surgery was performed. A detailed assessment of comorbidities was performed using several scales: Charlson and age adjusted Charlson score, physiological score of POSSUM. Postoperative complications were graded according to the Dindo-Clavien classification. RESULTS: During a 9 years period surgery was performed in 50 cases, 33 by TT approach and 17 by $\mathrm{TH}$ approach respectively. The mean age was $58.7 \pm 2.21$ years old (95\% CI 56.3-61.2). Patients in the TH group had a higher Charlson score (3 vs 2, $P=0.01$ ), age adjusted Charlson score (5 vs $4, P=0.03$ ) and physiological score (17 vs $15, P=0.04)$. TT techniques were mainly used for middle oesophageal cancers $(69.7 \%)$ and $\mathrm{TH}$ for lower oesophageal tumors $(82.4 \%)$. The overall operative morbidity was $60 \%$ with no difference between the two groups even for minor and major complications. Pulmonary complications occurred in 23 cases (46\%), cardiac complications in 5 cases (10\%), anastomotic leakage in 6 cases $(12 \%)$ and recurrent nerve paralysis in 6 cases (12\%). Multivariate analysis showed that age adjusted Charlson score $(\mathrm{OR}=2.77$; 95\%CI 1.114-6.9236 $)$ and physiological score $(\mathrm{OR}=1.7601 ; 95 \%$ CI 1.2067-2.5674) were predictors for complications. CONCLUSION: In our study mortality and morbidity showed no statistical difference in relation to the surgical approach. An accurate preoperative assessment and tailoring an adequate surgical approach can limit the percentage of postoperative complications.
\end{abstract}

KEY WORDS: ESOPHAGUS; ESOPHAGEAL CANCER; SURGICAL TREATMENT; TRANSTHORACIC APPROACH; TRANSHIATAL APPROACH

SHORT TITLE: Transthoracic versus transhiatal esophagectomy

HOW TO CITE: Filip B, Huţanu I, Croitoru C, Aniţei MG, Radu I, Scripcariu V. [Transthoracic versus transhiatal esophagectomy: comparative study regarding surgical approach in esophageal cancer] Jurnalul de chirurgie (Iaşi). 2013; 9(4): 325-333. DOI: 10.7438/1584-9341-9-4-4.

\section{BACKGROUND}

Esophageal cancer is the seventh cancer related deaths with 482,300 new cases per year [1] and usually occurs as either squamous cell carcinoma usually in the middle and upper part of the mediastinum or adenocarcinoma in the lower third and the gastroesophageal junction.

Received date: 16.09 .2013

Accepted date: 18.10 .2013

Correspondence to: Bogdan Filip MD

First Surgical Oncology Unit, Regional Institute of Oncology Iaşi

General Henry Mathias Berthlot Street, No. 2-4, 700483, Iaşi, Romania

Phone: 0040 (0) 745253149

E-mail: bfilip79@yahoo.com 
There has been a shift in the incidence of the histological subtypes of oesophageal cancer with the incidence of adenocarcinoma rising in the past 3 decades. The greatest recent changes in the management of oesophageal cancer are related to the treatment and surveillance of Barrett esophagitis and management of early stages oesophageal cancers. For locally advanced tumors surgery alone provides poor prognosis [2] and in these cases induction therapy is often used with the aim of increasing the $\mathrm{R}_{0}$ resections rate, treatment of micrometastasis and improvement of survival. Surgical resection provides the best survival advantage for early stages and in tumors with good response after neoadjuvant treatment. The type of surgical approach varies depending on surgeon and institutional preference; the two most utilised techniques are the transthoracic with cervical or mediastinal anastomosis and transhiatal techniques. The reference and most cited largest randomised trial comparing the two surgical approaches included 220 patients with adenocarcinoma who were assigned to either transhiatal resection or Ivor Lewis technique [3,4]. The transhiatal group had shorter hospitalisation (19 vs 15 days), less blood loss and less overall morbidity (57 vs $27 \%, P<0.001$ ). In the other group more lymph nodes were resected (31 vs $16, P<0.001)$ with no difference in terms of $\mathrm{R} 0$ resection rate, postoperative mortality ( 4 vs $2 \%, P=0.4$ ) and survival. The overall morbidity rate after esophagectomy ranges between 50 to $60 \%$ $[5,6]$, the most common being the cardiopulmonary complications.

The aim of our study was to compare the two major approaches for oesophagectomy in a group of patients operated for oesophageal cancer in terms of preoperative assessment and early surgical outcome.

\section{MATERIAL AND METHOD}

We performed an observational study analysing all the patients who underwent esophageal resections for oesophageal cancer, from January 2004 until March 2013. Tumors of the cervical oesophagus as well as subcardial tumors (Siewert III type) were excluded from this analysis.

Preoperative workout consisted in medical history and examination, functional evaluation, barium contrast esophagogram, endoscopy with biopsy, thorax and abdominal CT scan, abdominal ultrasound. Functional status was assessed by means of routine blood tests, electrocardiogram and pulmonary function tests. Second line investigations such as: cardiac ultrasound and cardiopulmonary stress tests were performed when necessary.

After initial diagnosis and staging, patients with locally advanced tumors were submitted to a neoadjuvant protocol according to the functional status.

Comorbidities have been quantified accordingly to the Charlson comorbidity scale [7] and age adjusted Charlson comorbidity scale [8] and included: hypertension (systolic blood pressure higher than 140 and/or diastolic pressure higher than 90); cardiac dysfunction (history of ischemic heart disease, unstable angina, cardiac failure, atrial fibrillation), pulmonary dysfunction $\left(\mathrm{FEV}_{1}<70 \%\right.$ of predicted values and / or vital capacity < 80\%), vascular dysfunction (history of abdominal aortic aneurism, vascular bypass, carotid stenosis), neurological dysfunction (history of cerebrovascular accident or transitory ischemic attack, epilepsy or Parkinson), liver cirrhosis, chronic renal failure, diabetes mellitus on treatment. In order to have a more accurate information regarding functional status of the patients we calculated the physiological score of the POSSUM (Physiological and Operative Severity Score for the enUmeration of Mortality and morbidity) based on the original description and codification of each component [9].

The POSSUM score was developed to allow risk-adjusted prediction of postoperative mortality and morbidity in surgery according to the physiological condition of the patients, severity of surgery, 
age and general health and consists of a physiological part (12 factors with 4 grades score) and an operative part (6 factors with 4 grades).

\section{Surgical procedures:}

\section{Transhiatal approach}

The patient was placed in the supine position with the head turned toward the right and the neck extended. After laparotomy, the stomach was mobilised by dividing the gastrocolic ligament while preserving the right gastroepiploic vessels.

The short gastric vessels were divided. The esophagogastric junction was freed from the hiatus; the lesser omentum and the gastrohepatic ligament were divided. The left gastric artery was ligated and divided with celiac lymph nodes dissected. The oesophagus was dissected under direct vision through the widened hiatus of the diaphragm up to the inferior pulmonary vein.

A left cervical incision is made along the anterior border of the left sterno-cleidomastoid muscle and dissection is carried out along the prevertebral space. When the cervical oesophagus was found, blunt dissection was performed down to the level of the thoracic outlet; blunt dissection was carried out along the oesophagus until the entire organ was mobilized. Several centimetres of oesophagus were gently pulled up into the cervical region and then transected.

The distal stump was carefully closed and the stomach and oesophagus were then removed through the diaphragmatic hiatus. After Kocher's manoeuvre and pyloroplasty, the oesophagus, cardia, and an area along the lesser curvature were resected and the stump was closed using a linear stapler or a running suture.

The stomach graft was delivered to the neck wound through the posterior mediastinal route by gentle traction using a silicone drain attached to the proximal part of the gastric tube and a hand sewn anastomosis was performed.

\section{Transthoracic approach}

Based on a surgeon's preference, when a triple approach was chosen, a right thoracotomy is performed as an initial step of the procedure and included removal of the azygous arch, low paratracheal, subcarinal, paraesophageal and parahiatal nodes in continuity with the resected esophagus. For tumors of the middle part of the thoracic oesophagus, the right reccurential lymph nodes were removed. The pleural cavity was drained and the chest wall was closed. Afterwards, the patient was placed in a supine position, in the same manner as for the transhiatal approach. The cervical and abdominal part of the intervention was similar to the transhiatal technique described above. This represents a modified McKeown technique; we prefer to perform thoracotomy as the first step to allow a better assessment of resectability in order to avoid potential unresectable locally advanced tumors. The stomach graft is pulled to the neck incision in similar fashion as in the transhiatal technique.

When the Ivor-Lewis technique is performed, the initial step was a median laparotomy with gastric mobilisation with preservation of the right gastric and gastroepiploic vessels and resection of the upper third of the stomach. After the abdominal stage, a right posterolateral thoracotomy is done allowing resection of the oesophagus and mediastinal lymphadenectomy. The intrathoracic anastomosis is performed manually.

In all patients a pyloroplasty and a jejunostomy feeding tube inserted at the end of surgery in order to provide accurate nutrition in the early postoperative period were performed. In patients with previous gastric surgery a colic interposition was preferred.

Pathologic examination was recorded in all patients and tumors were classified using the UICC TNM $7^{\text {th }}$ classification.

Postoperatively, patients were transferred to the intensive care unit and extubated when they were hemodinamically 
and respiratory stable; jejunostomy feeding was administered in the first postoperative day, swallow X-ray studies were performed on the 7 th postoperative day to assess the anastomosis and gastric tube passage and a liquid diet was initiated when there were no signs of leakage. Postoperative complications in any of the patients undergoing either of the two approaches were noted. Postoperative morbidity and mortality were defined as any complication or death which occurred within 30 days after surgery.

Complications were graded according to the Dindo Clavien classification [10] based on the therapeutic consequences. Grade I and II were considered minor complications and grade III and IV as major complications, grade V complications was considered any major complications leading to the death of the patient.
Based on these considerations, patients were divided in two groups: patients who underwent transhiatal approach (TH group) and respectively, patients who underwent transthoracic approach (TT group).

\section{Statistical analysis}

Statistical analysis was performed using MedCalc v 9.2.0.1. Medians and interquartile ranges at the $25^{\text {th }}$ and $75^{\text {th }}$ percentile were calculated and subsequently depicted when relevant. Mann-Whiney U tests and Chi-square testes were used when appropriate. Significance was set at $P<0.05$.

\section{RESULTS}

Between January 2004 and March 2013, fifty consecutive patients diagnosed with oesophageal cancer underwent surgical resections.

Table I Baseline characteristics

\begin{tabular}{lcccc}
\hline & TH $(n=17)$ & TT $(n=33)$ & Total $(n=50)$ & $\begin{array}{c}P- \\
\text { value }\end{array}$ \\
\hline Age & $60 \pm 1.48(51.6-67.68)$ & $57 \pm 2.17(54-62)$ & $58.7 \pm 2.21(56.3-61.2)$ & 0.53 \\
Charlson score (CS) & $3(2-4)$ & $2(2-3)$ & $3(2-4)$ & $\mathbf{0 . 0 1}$ \\
Age adjusted CS & $5(3.75-6.25)$ & $4(3-5)$ & $4(2-7)$ & $\mathbf{0 . 0 3}$ \\
POSSUM & $17(15.75-18.25)$ & $15(14-17.25)$ & $16(15.23-16.64)$ & $\mathbf{0 . 0 4}$ \\
Cardiac & $7(41.2 \%)$ & $8(24.2 \%)$ & $15(30 \%)$ & 0.13 \\
Pulmonary & $9(52.9 \%)$ & $10(30.3 \%)$ & $19(38 \%)$ & - \\
Renal & $1(5.9 \%)$ & 0 & $1(2 \%)$ & $\mathbf{0 . 0 4}$ \\
Diabetes & $4(23.5 \%)$ & $1(3 \%)$ & $5(10 \%)$ & $\mathbf{0 . 0 4}$ \\
Liver disease & $4(23.5 \%)$ & $1(3 \%)$ & $34(68 \%)$ & \\
ASA score & $8(47 \%)$ & $26(78.8)$ & $16(32 \%)$ & $\mathbf{0 . 0 2}$ \\
2 & $9(53 \%)$ & $7(21.2 \%)$ & $6(12 \%)$ & \\
3 & $2(11.7 \%)$ & $4(12.1 \%)$ & $10(20 \%)$ & 0.46 \\
Radiotherapy & $2(11.7 \%))$ & $8(24.2 \%)$ & $8(16 \%)$ & 0.69 \\
Chemotherapy & $2(11.7 \%)$ & $6(18.2 \%)$ & $11.89 \pm 0.44(11.4-12.4)$ & 0.16 \\
Radio-chemotherapy & $12 \pm 1.46(10.86-13.46)$ & $11.3 \pm 0.9(10.84-12.26)$ & $8440 \pm 456(7743-9136)$ & 0.36 \\
Hemoglobin & $8044 \pm 548(6484-9419)$ & $8640 \pm 444(7417-9944)$ & & \\
White Blood Cells & & & & \\
\hline
\end{tabular}

Patients' characteristics are summarised in Table I. There were no significant differences between the groups in terms of age. All of the patients were considered on preoperative assessment by the anaesthesiologist of being ASA 2 or 3 .
All scores which included a thoroughly assessment of the physiological status or of the co-existing comorbidities were statistically significant between the two subgroups of patients. Patients in which thoracotomy was performed had a better 
Charlson score $(P=0.01)$, age adjusted Charlson score $(P=0.03)$ and a better physiological score of POSSUM $(P=0.04)$. In terms of pre-existing conditions patients in which thoracotomy was performed had a lower incidence of cardiac, pulmonary, liver diseases and diabetes.

There was no difference in terms of neoadjuvant treatment, although there was a tendency for performing the induction therapy on the patients from the last 5 years included in the study.

The pathological characteristics of all the patients are summarised in Table II. In the vast majority of cases the tumor was localised in the middle and lower part of the esophagus, tumors considered Siewert I and II were encountered in $14 \%$ of all cases $(n=7)$. There were differences in terms of surgical approach based on tumor site: the transthoracic approach was more frequent used for tumors located in the middle third of the oesophagus and the transthoracic approach, for tumors located in the lower part of the oesophagus. The predominant histology $(72 \%, n=36)$ was squamous cell carcinoma. As a rule, tubulised stomach was used for reconstruction, in 6 cases it was used colon interposition graft due to the previous gastric surgery. In 44 cases anastomosis was performed in the neck, the remaining 6 cases being intrathoracic anastomosis, all of the cases were hand sewn anastomosis. Final pathological staging showed a predominance of the locally advanced tumors (stage III, 52\%, $n=26$ ), with no difference in terms of surgical approach. The patients were usually transferred intubated in the Intensive Care Unit and were extubated the next day.

Postoperative morbidity occurred in $60 \%$ of the cases $(n=30)$ and was mostly represented by cardiac and pulmonary complications (Table III). Eighteen patients developed minor complications according to Dindo-Clavien classification and 15 developed major complications. There were no differences in terms of overall, minor or major complications between the two subgroups of patients. Postoperative mortality rate was $10 \%$, with no difference based on surgical approach. In these cases death was due to the: sepsis secondary to anastomotic leakage, bilateral bronchopneumonia, massive myocardial infarction, stroke and severe pancreatitis. Mean hospital stay was $14 \pm 1.06$ days and ICU stay $6 \pm 0.55$ days with no difference between surgical approaches. Multivariate analysis as seen in Table IV showed that age adjusted Charlson score and physiologic score of POSSUM were independent prognostic factors affecting postoperative complications.

Table II Tumor and reconstruction characteristics

\begin{tabular}{|c|c|c|c|c|}
\hline & $\mathrm{TH}(n=17)$ & $\mathrm{TT}(n=33)$ & Total $(n=50)$ & $P$-value \\
\hline \multicolumn{5}{|l|}{ Tumor site } \\
\hline Thoracic esophagus & $3(17.6 \%)$ & $23(69.7 \%)$ & $26(52 \%)$ & \multirow{2}{*}{0.0007} \\
\hline Inferior esophagus & $14(82.4 \%)$ & $10(30.3 \%)$ & $24(48 \%)$ & \\
\hline \multicolumn{5}{|l|}{ Histology } \\
\hline SCC & $10(58.8 \%)$ & $26(78.8 \%)$ & $36(72 \%)$ & \multirow{2}{*}{0.18} \\
\hline $\mathrm{ADK}$ & $7(41.2 \%)$ & $7(21.2 \%)$ & $14(28 \%)$ & \\
\hline \multicolumn{5}{|l|}{ Stage } \\
\hline 1 & $4(23.5 \%)$ & $3(9 \%)$ & $7(14 \%)$ & \multirow{3}{*}{0.67} \\
\hline 2 & $4(23.5 \%)$ & $13(39.5 \%)$ & $17(34 \%)$ & \\
\hline 3 & $9(53 \%)$ & $17(51.5 \%)$ & $26(52 \%)$ & \\
\hline \multicolumn{5}{|l|}{ Reconstruction } \\
\hline Gastric graft & $15(88.2 \%)$ & $29(87.9 \%)$ & $44(88 \%)$ & \multirow{2}{*}{0.85} \\
\hline Colon interposition & $2(11.8 \%)$ & $4(12.1 \%)$ & $6(12 \%)$ & \\
\hline
\end{tabular}

SCC squamous cell carcinoma; ADK adenocarcinoma 


\section{DISCUSSION}

Despite the recent shift in management of oesophageal cancer to perform neoadjuvant chemo-radiotherapy, surgical resection remains the most important part of multimodal treatment for early stages and locally advanced disease [11]. The best surgical approach, in terms of outcomes and quality of lymphadenectomy is still a subject of debate. Although the minimally invasive techniques recently developed are used, the traditional surgical approaches are still the most used in surgical practice.

Several variables as comorbidities, tumoral stage, surgical technique (especially the type of approach) and surgeon and hospital experience should be considered when an audit of operative outcomes is performed.

A multicentre study regarding the preferred surgical techniques in oesophageal cancer [12] showed that the most common approaches are the transhiatal (35-44\%) and Ivor Lewis (36-50\%) with variability regarding the site of the tumor. In a small number of cases "en bloc" resection (3.9$6.5 \%$ ) and the three fields lymphadenectomy (13\% for proximal tumors and $1.3 \%$ for gastroesophageal junction tumors) were preferred.

Table III Outcomes after surgery

\begin{tabular}{lcccc}
\hline & TH $(n=17)$ & TT $(n=33)$ & Total $(n=50)$ & $P$-value \\
\hline Overall morbidity & $10(58.8 \%)$ & $20(60.6 \%)$ & $30(60 \%)$ & 0.99 \\
Pulmonary & $8(47 \%)$ & $15(45.4 \%)$ & $23(46 \%)$ & 0.99 \\
Cardiac & $1(5.9 \%)$ & $4(12.1 \%)$ & $5(10 \%)$ & 0.64 \\
Fistula & $2(11.8 \%)$ & $4(12.1 \%)$ & $6(12 \%)$ & 0.99 \\
Haemorrhage & $2(11.8 \%)$ & $3(9.1 \%)$ & $5(10 \%)$ & 0.99 \\
Neurologic & $1(5.9 \%)$ & $1(3 \%)$ & $2(4 \%)$ & 0.99 \\
Hepatic failure & - & $1(3 \%)$ & $1(2 \%)$ & - \\
Sepsis & $2(11.8 \%)$ & $8(24.2 \%)$ & $10(20 \%)$ & 0.46 \\
Recurrent palsy & $5(29.4 \%)$ & $2(6 \%)$ & $6(12 \%)$ & $\mathbf{0 . 0 3 7}$ \\
Minor complications & $6(35.2 \%)$ & $12(36.3 \%)$ & $18(36 \%)$ & 0.95 \\
Major complications & $4(23.5 \%)$ & $11(33.3 \%)$ & $5(30 \%)$ & 0.53 \\
Mortality & $1(5.9 \%)$ & $4(12.1 \%)$ & $6 \pm 0.55(5-7)$ & 0.64 \\
ICU stay & $6 \pm 1.15(4.6-8.8)$ & $6 \pm 0.62(5-7)$ & $14 \pm 1.06(12-16)$ & 0.70 \\
Hospital stay & $14 \pm 1.58(10.6-20.2)$ & $14 \pm 1.29(12-17.4)$ & & \\
\hline
\end{tabular}

Table IV Multivariate analysis for postoperative complications

\begin{tabular}{lccc}
\hline Variable & Odds Ratio $(\mathrm{OR})$ & $95 \%$ CI & $P$-value \\
\hline Age adjusted Charlson score & 2.7772 & $1.1140-6.9236$ & $\mathbf{0 . 0 2 8}$ \\
Charlson score & 0.3491 & $0.0865-1.4090$ & 0.139 \\
ASA & 1.7347 & $0.2202-13.6674$ & 0.60 \\
Physiologic score POSSUM & 1.7601 & $1.2067-2.5674$ & $\mathbf{0 . 0 0 3 3}$ \\
Thoracotomy & 1.2469 & $1.2067-2.5674$ & 0.80 \\
Tumor site & 0.6258 & $0.1284-3.0504$ & 0.5619 \\
\hline
\end{tabular}

Te prognostic scores based on general evaluation of the patient (ASA score) and on the overall comorbidities (POSSUM, Charlson and age adjusted Charlson scores) were significantly different in the two groups of patients (Table I). This is probably explained by the selection of patients suitable for a transthoracic resection; 
basically, the thoracotomy was not performed in a patient with an impaired cardiac and pulmonary function and this was directly reflected in the scores for functional assessment.

Age did not differ in the two groups, result which is concurent with literature data [13]. Comorbidities and overall functional capacity are more likely to affect perioperative morbidity more than age alone [14]. Patients with poor pulmonary status (forced vital capacity of less than $80 \%$ and forced expiratory volume in one second less than $70 \%$ of predicted value) have been associated with higher risk for pulmonary complications [15].

In our series patients suffering from an impaired pulmonary function were not considerate suitable for thoracotomy, and a transhiatal approach was used.

Other organ dysfunction can increase the risk of postoperative morbidity and mortality.

Liver cirrhosis can be a contraindication for surgery in some centres although Child A cirrhotic patient can undergo esophagectomy with acceptable mortality and morbidity [16]. All the patients in our series with a preoperative impaired liver function developped a major complication and death occurred in two cases, no matter the type of surgical approach.

Based on the results of a metaanalysis, the mortality rate after esophagectomy varies from 0 to $22 \%$ depending on the selection of the patients, experience of surgical team and the level of postoperative care [17]. Low volume centres (less than 5 cases per year) had a median of $18 \%$ mortality and high-volume centres (more than 20 cases per year) a median $4.9 \%$ mortality. We had a $10 \%$ mortality rate by performing 5 to 6 oesophagectomies per year.

Pulmonary complications are the most frequent complication after esophagectomy and have been the leading cause of twothirds of postoperative mortality [18]. In a 10 year review of 1,777 oesophagectomies it was reported a $49.5 \%$ rate of complications of which pneumonia represented $21.4 \%$, respiratory failure which required reintubation in $16.2 \%$ [5]. The choice of approach may also have a direct impact on respiratory complications. In a randomised study of 220 patients to a transthoracic versus a transhiatal approach for mid to distal oesophageal cancer, the comparison revealed a significantly higher rate of pulmonary complications in the transthoracic group $(57 \%$ vs $27 \%$, $P<0.001)$, but with the same 5-year survival [3].

In our study we noted similar rates of pulomary complications. Given the study type (observational) we cannot conclude that both surgical approaches are similar in term of pulmonary morbidity rate. However we can conclude that a tailoring surgical approach can decrease the pulomonary complications.

Cardiovascular complications after esophagectomy includes a large group of events, in which atrial fibrillation remains the most common, with reported rates from $11.5 \%$ until $22 \%[6,19]$. It is very important to prevent this type of complication because of the concern that conduit perfusion can be altered in the periods of relative hypotension precipitated by atrial fibrillation. In our series we encountered atrial fibrillation as a direct effect of another major complication, such as sepsis following anastomotic leak.

Recurrent nerve injury occurs during dissection of the cervical oesophagus and it is associated with the site of the anastomosis. The results of a meta-analysis [20] showed that the cervical anastomosis increases this risk $(\mathrm{OR}=7.14$; 95\%CI: 1.75 29.14; $P=0.006$ ). In our series we found to have significantly higher rates of recurrent nerve injury following the transhiatal approach, although the major part of the anastomosis was performed in the cervical region.

One of the most challenging steps of the esophagectomy is the preservation of an accurate perfusion at the site of the anastomosis; compromised conduit 
perfusion can lead to a reduced blood flow with an impaired healing and a high risk for fistula [21]. This is important when performing a cervical anastomosis that requires a longer gastric conduit with a high risk of diminished blood flow. The anastomotic leakage rates are reported in the literature from $10.9 \%$ to $16 \%$ [3,6,22,23]. A large study [24] reported a $2 \%$ rate of gastric tip necrosis that required reintervention. The endoscopic evaluation of oesophageal replacement revealed gastric conduit ischemia in $9.2 \%$ of patients [23]. The thoracic anastomosis requires a shorter conduit length and less tension which are the key elements for a lower leak rate. The disadvantages of this site of the anastomosis are the opening of thoracic cavity and the possible consequences of an intrathoracic fistula. Although in our series there were no differences in terms of surgical approach we encountered only one fistula by performing an intrathoracic anastomosis, all the others were cervical site fistulas after either transhiatal or McKeown techniques. So, the choice of anastomotic location remains surgeon dependent based on personal experience and awareness of outcomes.

Our study has several drawbacks. The first one is that represents a small number of patients and this can limit the accuracy of the results. This is the direct result of relatively low percentage of patients suitable for surgery in Romania; many of the newly diagnosed cases are locally advanced or metastatic lesions in a patient with severe comorbid conditions. The second major drawback is the heterogeneous cohort of patients, we had a relatively long inclusion period and this induced a bias in the appliance of multimodal treatment, perioperative management in terms of early extubation, fluid restriction management, pain management and respiratory rehabilitation. Also, the management of postoperative complications was relatively different during the inclusion period, with a higher clinical suspicion toward the occurrence of a complication after even a minor change in the postoperative course of a patient.

\section{CONCLUSIONS}

Surgery is still the standard of care and the best way to cure or palliate patients with oesophageal cancer. Significant improvement in oncologic staging and functional preoperative evaluation has led to better patient selection and better outcomes. Despite these improvements, esophagectomy is still associated with high morbidity and mortality risks. In order to minimalize the surgical trauma to the patient a various surgical approaches are used to treat patients. The surgeon should put in balance the oncologic principles of surgery (complete removal of the tumor with negative longitudinal and circumferential margins and adequate lymph node removal) and the risks of a specific surgical approach. The best way to reduce the surgical impact is to personalise the surgical approach to patient and tumor characteristics.

\section{CONFLICT OF INTERESTS}

None to declare.

\section{REFERENCES}

1. Jemal A, Bray F, Center MM, Ferlay J, et al. Global cancer statistics. CA: a cancer journal for clinicians. 2011;61(2): 69-90.

2. Malthaner RA, Wong RK, Rumble RB, et al. Neoadjuvant or adjuvant therapy for resectable esophageal cancer: a systematic review and meta-analysis. BMC Med. 2004; 2: 35.

3. Hulscher JB, van Sandick JW, de Boer AG, et al. Extended transthoracic resection compared with limited transhiatal resection for adenocarcinoma of the esophagus. $N$ Engl J Med. 2002; 347(21): 1662-1669.

4. Omloo JM, Lagarde SM, Hulscher JB, et al. Extended transthoracic resection compared with limited transhiatal resection for adenocarcinoma of the $\mathrm{mid} /$ distal esophagus: five-year survival of a randomized clinical trial. Ann Surg. 2007; 246(6): 992-1000.

5. Bailey SH, Bull DA, Harpole DH, et al. Outcomes after esophagectomy: a ten-year prospective cohort. Ann Thorac Surg. 2003; 75(1): 217-222. 
6. Seely AJ, Ivanovic J, Threader $J$, et al. Systematic classification of morbidity and mortality after thoracic surgery. Ann Thorac Surg. 2010; 90(3): 936-942.

7. Charlson ME, Pompei P, Ales KL, et al. A new method of classifying prognostic comorbidity in longitudinal studies: development and validation. J Chronic Dis. 1987; 40(5): 373-383.

8. Charlson M, Szatrowski TP, Peterson J, et al. Validation of a combined comorbidity index. $J$ Clin Epidemiol. 1994; 47(11): 1245-1251.

9. Copeland GP, Jones D, Walters M. POSSUM: a scoring system for surgical audit. Br J Surg. 1991; 78(3): 355-360.

10. Dindo D, Demartines N, Clavien PA. Classification of surgical complications: a new proposal with evaluation in a cohort of 6336 patients and results of a survey. Ann Surg. 2004; 240(2): 205-213.

11. DeMeester SR. Adenocarcinoma of the esophagus and cardia: a review of the disease and its treatment. Ann Surg Oncol. 2006; 13(1): 12-30.

12. Enestvedt CK, Perry KA, Kim C, et al. Trends in the management of esophageal carcinoma based on provider volume: treatment practices of 618 esophageal surgeons. Dis Esophagus. 2010; 23(2): 136-144.

13. Internullo E, Moons $J$, Nafteux $P$, et al. Outcome after esophagectomy for cancer of the esophagus and GEJ in patients aged over 75 years. Eur J Cardiothorac Surg. 2008; 33(6): 1096-10104.

14. Markar SR, Karthikesalingam A, Thrumurthy $\mathrm{S}$, et al. Systematic review and pooled analysis assessing the association between elderly age and outcome following surgical resection of esophageal malignancy. Dis Esophagus. 2013; 26(3): 250-262.

15. Kuwano H, Sumiyoshi K, Sonoda K, et al. Relationship between preoperative assessment of organ function and postoperative morbidity in patients with oesophageal cancer. Eur $J$ Surg. 1998; 164(8): 581-586.
16. Lu MS, Liu YH, Wu YC, et al. Is it safe to perform esophagectomy in esophageal cancer patients combined with liver cirrhosis? Interact Cardiovasc Thorac Surg. 2005; 4(5): 423-425.

17. Metzger R, Bollschweiler E, Vallbohmer D, et al. High volume centers for esophagectomy: what is the number needed to achieve low postoperative mortality? Dis Esophagus. 2004; 17(4): 310-314.

18. Atkins BZ, Shah AS, Hutcheson KA, et al. Reducing hospital morbidity and mortality following esophagectomy. Ann Thorac Surg. 2004; 78(4): 1170-1176.

19. Murthy SC, Law S, Whooley BP, et al. Atrial fibrillation after esophagectomy is a marker for postoperative morbidity and mortality. $J$ Thorac Cardiovasc Surg. 2003; 126(4): 11621167.

20. Biere SS, Maas KW, Cuesta MA, et al. Cervical or thoracic anastomosis after esophagectomy for cancer: a systematic review and meta-analysis. Dig Surg. 2011; 28(1): 29-35.

21. Boyle NH, Pearce A, Hunter D, et al. Intraoperative scanning laser Doppler flowmetry in the assessment of gastric tube perfusion during esophageal resection. $J \mathrm{Am}$ Coll Surg. 1999; 188(5): 498-502.

22. Orringer MB, Marshall B, Chang AC, et al. Two thousand transhiatal esophagectomies: changing trends, lessons learned. Ann Surg. 2007; 246(3): 363-372.

23. Briel JW, Tamhankar AP, Hagen JA, et al. Prevalence and risk factors for ischemia, leak, and stricture of esophageal anastomosis: gastric pull-up versus colon interposition. $J$ Am Coll Surg. 2004; 198(4): 536-541.

24. Urschel JD, Urschel DM, Miller JD, et al. A meta-analysis of randomized controlled trials of route of reconstruction after esophagectomy for cancer. Am J Surg. 2001; 182(5): 470-475. 
